\title{
Skin rash in the intensive care unit: Stevens-Johnson syndrome, toxic epidermal necrolysis, or a rare manifestation of a hidden cutaneous malignancy: A case report
}

\author{
FARAH AL-SAFFAR ${ }^{1}$, SAIF IBRAHIM ${ }^{1}$, PUJAN PATEL $^{1}$, RAFIK JACOB $^{1}$, CARLOS PALACIO $^{1}$ and JAMES CURY ${ }^{2}$ \\ ${ }^{1}$ Department of Internal Medicine; ${ }^{2}$ Division of Pulmonary and Critical Care Medicine, \\ Department of Internal Medicine, University of Florida-Jacksonville, Jacksonville, FL 32209, USA
}

Received August 12, 2015; Accepted November 30, 2015

DOI: $10.3892 / \operatorname{mco} .2015 .713$

\begin{abstract}
Skin rashes are infrequently encountered in the intensive care units, either as a result or as a cause of admission. The entities of Stevens-Johnson syndrome (SJS) and toxic epidermal necrolysis (TEN) form a spectrum of desquamating skin diseases that have multiple etiologies, the most common being drug-related reactions; very rarely, the cause may be cutaneous malignancies. We herein present a unique case of a 54-year-old male patient with psoriasis treated with methotrexate, who presented with a cellulitis-like clinical picture, then developed a severe progressive systemic inflammatory response syndrome, and progressed clinically to SJS, then TEN even after discontinuing the antibiotics and methotrexate. A skin biopsy demonstrated an aggressive and rapidly-progressing T-cell lymphoma. The present case highlights the necessity of skin biopsy when encountering SJS and TEN in the ICU in order to identify potentially treatable/controllable causes. Although it appeared reasonable to correlate TEN solely to medications, the skin biopsies clearly demonstrated an aggressive T-cell skin lymphoma. In a patient with a better general condition it may have been helpful to treat this malignancy. TEN is a life-threatening condition and skin biopsy is the cornerstone of diagnosis, despite the presence of multiple risk factors and the typical physical findings of a drug-induced reaction.
\end{abstract}

Correspondence to: Dr Farah Al-Saffar, Department of Internal Medicine, University of Florida-Jacksonville, 651-3W 8th Street, L-18, Jacksonville, FL 32209, USA

E-mail: farah.al-saffar@jax.ufl.edu

Abbreviations: ICU, intensive care unit; CT, computed tomography; MTX, methotrexate; SJS, Stevens-Johnson syndrome; TEN, toxic epidermal necrolysis

Key words: toxic epidermal necrolysis, Stevens-Johnson syndrome, lymphoma, cutaneous lymphoma

\section{Introduction}

Toxic epidermal necrolysis (TEN) is a progressive extensive skin disease that is potentially fatal. TEN is defined by formation of bullae and skin sloughing, with purpuric spots covering $\geq 30 \%$ of the body surface area, or skin sloughing and bullae alone, involving $10 \%$ of the body surface area. The skin manifestations are associated with a marked systemic inflammatory response syndrome and major electrolyte disturbances, with a mortality rate of $>30 \%$ (1). TEN is classically medication-induced. A skin biopsy is essential for diagnosis and exclusion of other diseases masquerading as or coexisting with TEN. We herein describe a case where skin biopsy in a patient with classical TEN revealed a cutaneous T-cell lymphoma complicated by TEN. To the best of our knowledge, very few cases of TEN associated with cutaneous T-cell lymphoma have been reported to date (2-4).

\section{Case report}

A 54-year-old African-American man with a past medical history of psoriasis diagnosed 6 years prior, diabetes mellitus, chronic pancreatitis, heart failure and hypertension, presented to the emergency department for evaluation of fever $\left(101.7^{\circ} \mathrm{F}\right.$ for 3 days) accompanied by chills, body aches, pruritus and edema. The patient also reported recent prolonged sun exposure and a progressive scaly rash on his face and neck that had first appeared 3 weeks prior to presentation. The patient attributed his skin rash to methotrexate (MTX) and denied any recent sick contacts, chest pain, weight loss, vomiting, abdominal pain, dysuria, cough or headaches.

The patient's home medications included MTX and prednisone for psoriasis, insulin for diabetes, and lisinopril, metoprolol and aspirin for hypertension and heart failure. All medications were held on admission. In the emergency department, the patient's vitals included an elevated oral temperature of $104.4^{\circ} \mathrm{F}$, a heart rate of 168 beats $/ \mathrm{min}$, blood pressure $89 / 70 \mathrm{mmHg}$ and a glucose level of $138 \mathrm{mg} / \mathrm{dl}$. On physical examination, the patient was in moderate distress; his lungs were clear and the cardiac sounds were normal, without any rubs or gallops, but he was tachycardic. The abdomen was soft, with active bowel sounds. The skin examination 


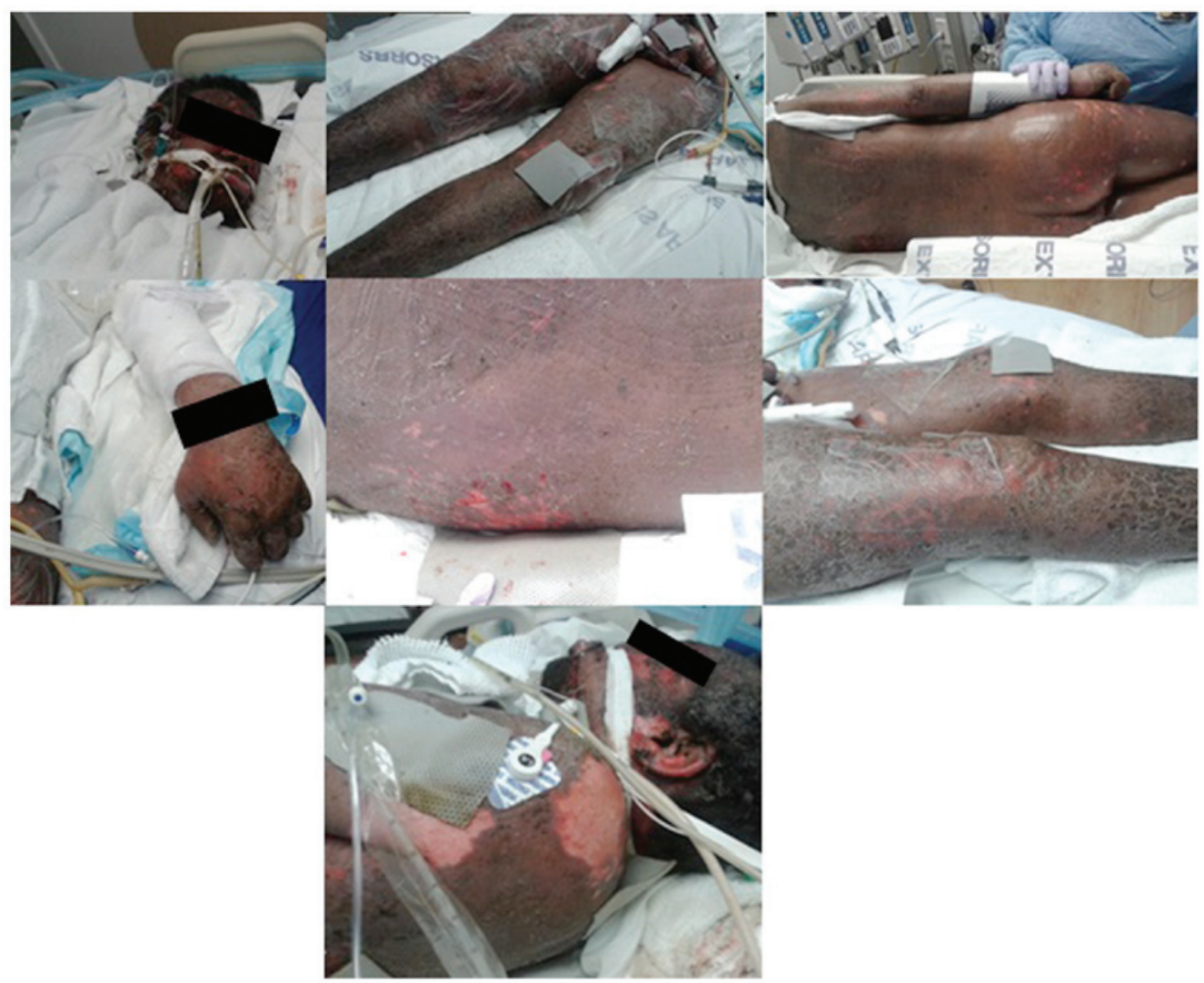

Figure 1. Extensive cutaneous sloughing indicative of toxic epidermal necrolysis.

A

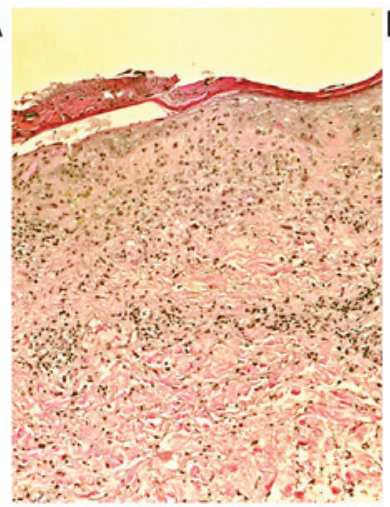

B

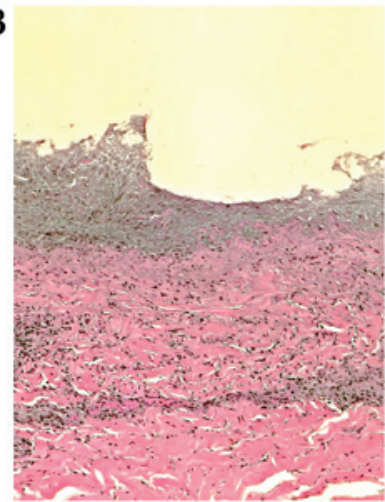

C

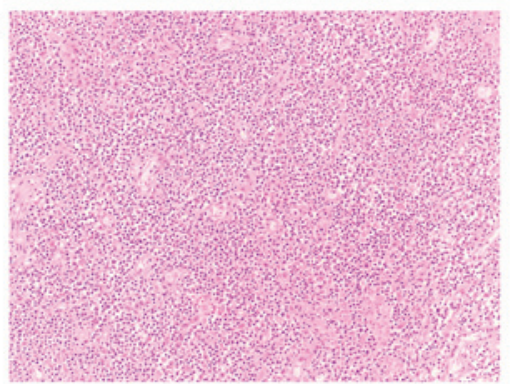

Figure 2. Skin biopsy. (A) Scale crust with frank epidermal necrosis and moderate lymphocytic infiltrate within the dermis. (B) Dermal fragment with denuded epidermis, moderate lymphocytic infiltrate and early collagen necrosis. (C) Pleomorphic nuclei with nucleomegaly and hyperchromasia.

revealed a dry scaly rash on the upper and lower extremities bilaterally. Erythema and pigmentation were observed on the face, without blisters. Palpable lymph nodes were detected

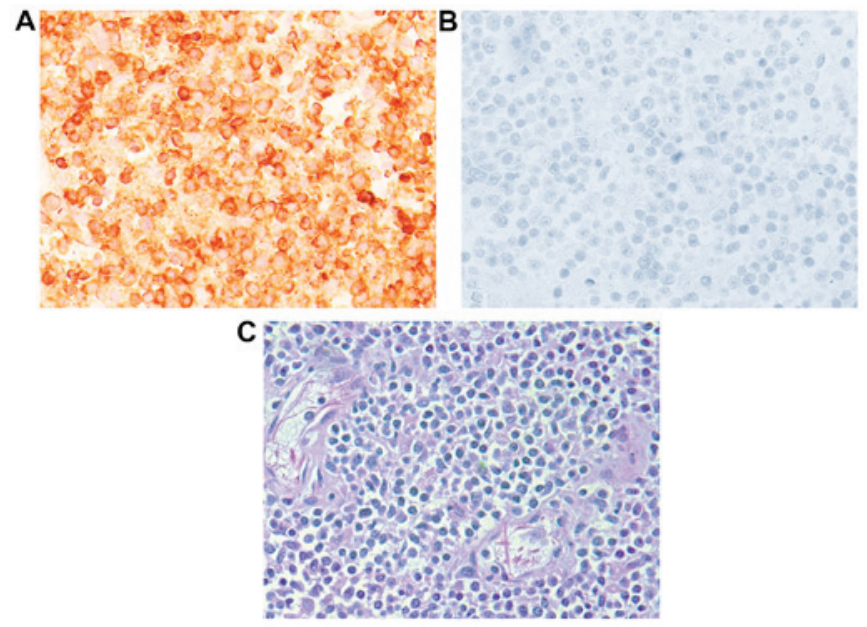

Figure 3. Lymph node biopsy. The lesional cells exhibited (A) strong, diffuse positivity for CD3 and (B) were negative for CD20. (C) Diffuse lymphoid infiltrate with effacement of lymph node architecture.

in the bilateral inguinal areas. The laboratory test results revealed leukocytosis $\left(20,900\right.$ cells $\left./ \mathrm{mm}^{3}\right)$, but were otherwise unremarkable.

The patient's chest X-ray was normal, but a computed tomography (CT) scan of his chest, abdomen and pelvis revealed bulky axillary lymphadenopathy and prominent mediastinal lymph nodes, with extensive retroperitoneal, external iliac chain and inguinal lymphadenopathy.

The patient was fluid-resuscitated; blood and urine cultures were obtained, and a lumbar puncture was performed prior 
to starting antibiotics, initially consisting of i.v. vancomycin and cefepime for empiric treatment of sepsis from unknown source. All the cultures were negative and the antibiotics were de-escalated to clindamycin to cover for cellulitis. Despite the negative cultures, the patient developed an altered mental status and underwent a repeat head CT and lumbar puncture, which were normal. At that time, antibiotic therapy was escalated again to i.v. vancomycin, piperacillin/tazobactam and tobramycin for refractory sepsis. The skin was biopsied and a bone marrow biopsy was performed for thrombocytopenia and hemolysis, with an elevated immature platelet count of $8.6 \%$.

Five days later, the patient developed respiratory failure, became tachypneic and tachycardic, with a significantly increased work of breathing, requiring intubation and mechanical ventilation. The skin rash became more desquamative; Stevens-Johson syndrome (SJS) was suspected and was attributed to MTX, which had been held on admission. The clinical picture progressed rapidly to TEN (Fig. 1).

Subsequently, the patient developed acute renal failure requiring hemodialysis. The prior skin biopsy was interpreted as being consistent with T-cell cutaneous lymphoma (Fig. 2). The peripheral blood smear was negative for Sezary cells. Subsequent blood cultures grew vancomycin-resistant enterococcus and candida at 15 days. The antibiotics were changed to fluconazole, daptomycin and meropenem.

The patient's skin rash progressively worsened and, on hospital day 23, it progressed to TEN. Fluconazole was changed to caspofungin, as it is associated with a lower incidence of TEN. Lymph node core needle biopsy was performed, the results of which were consistent with lymphoma (Fig. 3). Given the patient's clinical instability, he was not deemed to be a candidate for chemotherapy. The rash progressively became more extensive and the patient subsequently succumbed to his condition.

\section{Discussion}

SJS and TEN have long been viewed as a continuum with escalating severity, as the exfoliating pathology advances to involve larger body areas. Between those rare entities, SJS is 3-5 times more common compared with TEN, and usually encountered in women. Studies have shown that a dysregulated immune response, genetics and drugs may be predisposing risk factors to developing these two pathologies (5).

TEN is an entity frequently seen in the intensive care unit (ICU) and it is usually drug-induced. Thus, this was the initial impression when assessing the present case. Our patient was on MTX for presumed psoriasis. MTX has been found to be associated with TEN. However, the rash progressed from SJS to TEN despite discontinuing MTX. Alternatively, SJS/TEN could have been drug-induced, secondary to the antibiotics the patient received for sepsis. When reviewing the literature, no TEN cases were reported in association with daptomycin, only 4 with pippracillin-tazobactam (6-9), and 12 cases with fluconazole (10-18).

The fact that the patient presented with SJS progressing to TEN despite discontinuing MTX and despite being on antibiotics that have minimal association with TEN, makes the association with cutaneous T-cell lymphoma more likely. Lymphoma was confirmed by skin, bone marrow and lymph node biopsies that solely revealed T-cell lymphoma. There have been several reported cases of TEN in lymphoma patients with presentations similar to our case.

While skin rashes in the ICU are frequently encountered, it is important to perform skin biopsies, particularly when the patient's status progressively deteriorates. It is also important to rule out other causes of desquamating skin rashes, such as human immunodeficiency virus, pemphigoid skin disorders, drug-induced SJS and TEN and, rarely, cutaneous T-cell lymphoma.

A witnessed informed consent for publication of this case report was obtained from the patient's next of kin.

\section{References}

1. Pereira FA, Mudgil AV and Rosmarin DM: Toxic epidermal necrolysis. J Am Acad Dermatol 56: 181-200, 2007.

2. Schoeffler A, Levy E, Weinborn M, Cuny JF, Schmutz JL, Barbaud A, Cribier B and Bursztejn AC: Stevens-Johnson syndrome and Hodgkin's disease: A fortuitous association or paraneoplastic syndrome? Ann Dermatol Venereol 141: 134-140, 2014 (In French).

3. Jones B, Vun Y, Sabah M and Egan CA: Toxic epidermal necrolysis secondary to angioimmunoblastic T-cell lymphoma. Australas J Dermatol 46: 187-191, 2005.

4. Sadi AM, Toda T, Kiyuna M, Tamamoto T, Ohshiro K and Shinzato R: An autopsy case of malignant lymphoma with Lyell's syndrome. J Dermatol 22: 594-599, 1995.

5. Ellender RP, Peters CW, Albritton HL, Garcia AJ and Kaye AD: Clinical considerations for epidermal necrolysis. Ochsner J 14: 413-417, 2014.

6. Bajaj P, Prematta MJ and Ghaffari G: A sixty-five-year-old man with rash, fever, and generalized weakness. Allergy Asthma Proc 32: e1-e3, 2011.

7. Lam AI, Randhawa I and Klaustermeyer W: Cephalosporin induced toxic epidermal necrolysis and subsequent penicillin drug exanthem. Allergol Int 57: 281-284, 2008.

8. Craycraft ME, Arunakul VL and Humeniuk JM: Probable vancomycin-associated toxic epidermal necrolysis. Pharmacotherapy 25: 308-312, 2005.

9. Cheriyan S, Rosa RM and Patterson R: Stevens-Johnson syndrome presenting as intravenous line sepsis. Allergy Proc 16: 85-87, 1995.

10. Gussenhoven MJ, Haak A, Peereboom-Wynia JD and van 't Wout JW: Stevens-Johnson syndrome after fluconazole. Lancet 338: 120, 1991.

11. Islam S, Singer M and Kulhanjian JA: Toxic epidermal necrolysis in a neonate receiving fluconazole. J Perinatol 34: 792-794, 2014.

12. George J, Sharma A, Dixit R, Chhabra N and Sharma S: Toxic epidermal necrolysis caused by fluconazole in a patient with human immunodeficiency virus infection. J Pharmacol Pharmacother 3: 276-278, 2012.

13. Pasmatzi E, Monastirli A, Georgiou S, Sgouros G and Tsambaos D: Short-term and low-dose oral fluconazole treatment can cause Stevens-Johnson syndrome in HIV-negative patients. J Drugs Dermatol 10: 1360, 2011.

14. Thiyanaratnam J, Cohen PR and Powell S: Fluconazole-associated Stevens-Johnson syndrome. J Drugs Dermatol 9: 1272-1275, 2010.

15. Monastirli A, Pasmatzi E, Vryzaki E, Georgiou S and Tsambaos D: Fluconazole-induced Stevens-Johnson syndrome in a HIV-negative patient. Acta Derm Venereol 88: 521-522, 2008.

16. Ofoma UR and Chapnick EK: Fluconazole induced toxic epidermal necrolysis: A case report. Cases J 2: 9071, 2009.

17. Craythorne E and Creamer D: Stevens-Johnson syndrome due to prophylactic fluconazole in two patients with liver failure. Clin Exp Dermatol 34: e389-e390, 2009.

18. Azón-Masoliver A and Vilaplana J: Fluconazole-induced toxic epidermal necrolysis in a patient with human immunodeficiency virus infection. Dermatology 187: 268-269, 1993. 